\title{
Endovascular treatment for acute ischemic stroke: IVTPA VS IA TPA
}

\begin{abstract}
Treatment with intravenous tissue plasminogen activator (IV tPA) alone is associated with an estimated $40 \%$ efficacy in early recanalization of major coronary artery (MCA) occlusions. AIS still has almost a $60 \%$ rate of mortality or disability in patients treated with IV tPA, despite aggressive therapy. Endovascular treatment is currently being researched. The IA administration of tPA is safe and effective, but remains an unapproved, off-label treatment for AIS. Hypothesized advantages of intra-arterial tPA include direct visualization of acute large vessel thrombi; the ability to customize the tPA dose and location of drug delivery based on features of the individual clot, and potentially more complete thrombolysis. This paper exams the evidence on Intraarterial tPA administration versus IV tPA in patients outside of the $4.5 \mathrm{hr}$ treatment window established by the FDA.
\end{abstract}

Volume 3 Issue 2 - 2015

\author{
Leah Sheffer \\ University of Florida/Pipeline Rx, USA
}

Correspondence: Leah Sheffer, University of Florida/Pipeline Rx, 5600 N River Road, Suite 800, Rosemont, IL 600 I8, USA, Tel 3097244600,Email leahcsheffer@gmail.com

Received: April 27, 2015 | Published: December 21, 2015

Keywords: endovascular treatment, acute ischemic stroke, tpA

\begin{abstract}
Abbreviations: MCA, major coronary artery; CSC, comprehensive stroke centers; TJC, the joint commission; AIS, acute ischemic Stroke; MRI, magnetic resonance imaging; ICA, internal carotid arteries; MCA, middle cerebral arteries; MT, mechanical thromboectomies; CT, computerized tomography; NIHSS, national institutes of health stroke scale; MRS, modified rankin scores; WMH, white matter injury; DM, diabetes mellitus; WMD, white matter disease; TICI, thrombolysis in cerebral infarction scale
\end{abstract}

\section{Introduction}

Treatment with intravenous tissue Plasminogen activator (IV PA) alone is associated with an estimated $40 \%$ efficacy in early recanalization of major coronary artery (MCA) occlusions. The number of Primary Stroke Centers (PSC) and Comprehensive Stroke Centers (CSC) are growing across the country. The Joint Commission (TJC), Target Stroke, and Get with the Guidelines all promote safe and effective practice protocols in regards to the treatment of acute ischemic stroke (AIS) in the PSCs and CSCs. The NINDS trial established IV TPA as the current standard of care therapy based on safety and efficacy for AIS within 3hours of the onset of symptoms. ${ }^{1}$ The ECASS study expanded the treatment window from 3 hours to 4.5hours after the onset of AIS symptoms. ${ }^{2}$ Theoretically, IV tPA can be administered in any hospital when used appropriately. Over a decade has passed since the NINDS trial was published and AIS still has almost a $60 \%$ rate of mortality or disability in patients treated with IV tPA, despite aggressive therapy.

The goals of AIS treatment are recanalization of the occluded cerebral arteries and the reperfusion of penumbral and ischemic areas in order to decrease or prevent disability and mortality. An impending need to improve outcomes exists. Eligibility for IV tPA therapy is based on clinical presentation, the appearance of stroke on multimodal computerized tomography (CT) or magnetic resonance imaging (MRI) of the brain, and the time since the onset of symptoms, not on the visualization of the targeted thrombus. Potential causes of IV tPA treatment failure include low rates of recanalization of major occlusions $(14 \%$ in the internal carotid arteries [ICA] and
$55 \%$ in the middle cerebral arteries [MCA]) or reocclusion resulting from collateral failure, reperfusion injury, edema, or other unknown mechanisms, which lead to clinical deterioration following initial improvement. ${ }^{3}$ Recanalization is the most critical determinant of successful clinical outcomes. The PROACT II trial provided the first evidence for the effectiveness of endovascular treatment. ${ }^{4}$ This trial compared intra-arterial (IA) pro-urokinase to IV heparin at 6hours after the onset of AIS symptoms. The IA pro-urokinase increased the recanalization rate of MCA occlusions by $66 \%$ compared to $18 \%$ with IV heparin. Patient outcomes were improved. However, the successfulness of this trial is limited in clinical application. The Food and Drug Administration requires 2 trials to confirm the results for drug approval. The subsequent trial never took place due to cost and the ethics of a placebo controlled trial and questions were left unanswered. Pro-urokinase is no longer commercially available.

The focus on the strategy for effective occlusion recanalization shifted to the development and use of mechanical thromboectomies (MT). The FDA approved these devices under 510(k) clearance, not requiring evidence of efficacy, based off of uncontrolled trials that demonstrated recanalization of large-artery occlusions. ${ }^{5,6}$ FDA device clearance is an easier, streamlined process compared to drug approval.

The IA administration of tPA is safe and effective, but remains an unapproved, off-label treatment for AIS. Hypothesized advantages of IA tPA include direct visualization of acute large vessel thrombi; the ability to customize the IPA dose and location of drug delivery based on features of the individual clot, and potentially more complete thrombolysis. Recanalization can be visualized and confirmed during IA treatment as a marker of procedural success. The potential disadvantages to IA tPA are that it is still unproven as a superior monotherapy method of treatment, requires additional time to administer and coordinate compared to IV tPA, and requires the expertise of a neuro-interventionist at a CSC, usually not at a PSC or non-stroke hospital. Patient transfers need to be arranged and afterhours services or on-call staff need to be made available as time is ticking and the brain is rapidly deteriorating. The EMS trial is the 
pilot trial, which established the safety and efficacy of combined IV tPA and local IA tPA therapy for stroke within 3hours of the onset of symptoms. ${ }^{7}$ The IMS series of trials attempted to combine the advantages of the two routes of administration by comparing IV tPA alone to IV tPA followed by IA tPA. ${ }^{8-10}$ The theory behind the IV tPA followed by IA tPA method was to merge the improved time to initiation of therapy associated with IV tPA along with the more complete recanalization associated with IA tPA. The purpose of this paper is to determine if IA tPA has a place in the treatment of AIS based on the review of the three studies presented in Table $1.8,11,12$ These studies all compare standard treatment of AIS with IV tPA to endovascular therapy, defined as IA tPA bridged with IV tPA therapy with or without the use of MT.

\section{Evaluation of the studies}

The MR RESCUE trial was an eight year, randomized, controlled, open-labeled, blinded outcome, multicenter trial. ${ }^{11}$ There were 118 eligible patients enrolled in this trial that had a National Institutes of Health Stroke Scale (NIHSS) score between 6 and 29, large-vessel occlusion involving anterior circulation in the ICA and the M1 and M2 regions of the middle cerebral cortex, and favorable penumbral patterns in $58 \%$ of the patients. Favorable penumbral patterns are defined as an infarct core less than $90 \mathrm{~mL}$ and a total ischemic tissue volume, or core, as less than or equal to $70 \%$ (minimum mismatch of $30 \%$ ). The mean age of the patients was 65.5 years. The mean time to enrollment was 5.5 hours and treatment enrollment was required within 8hours from the onset of AIS symptoms. The control group received the standard of care treatment, consisting of tPA $0.9 \mathrm{mg} / \mathrm{kg}$ IV (10\% bolus; $90 \mathrm{mg}$ max) within 3hours from the onset of AIS symptoms. IV tPA was administered to $29.6 \%$ of the participants in this treatment arm. Patients with a persistent target occlusion, as shown on a MRA or CTA after standard treatment, were eligible for endovascular therapy if a favorable penumbral pattern was evident. IV tPA was initially administered to $43.8 \%$ of the participants in the endovascular treatment arm. The patients in the treatment group received up to $14 \mathrm{mg}$ of IA tPA within 6hours of onset as symptoms as rescue therapy in conjunction with first generation MT device use within 8 hours. The mean time to groin puncture was 6.2 hours. The mean dose of IA tPA administered was $5.1 \mathrm{mg}$. The event analysis was determined using a nonparametric two-way analysis of variance comparing the outcomes based on the modified Rankin Scores (mRS). The goal of the MR RESCUE trial is to compare the effectiveness of treating acute ischemic stroke with MT, using the Merci Retriever or the Penumbra System, within 8hours of symptom onset to standard medical treatment and to identify people who might benefit from MT by the appearance of stroke on CT or MR imaging. The IMS III trial is a randomized, controlled, multicenter trial, which compares standard IV tPA treatment to endovascular therapy after IV tPA in 656 patients. ${ }^{8}$ The mean age of the participants in the endovascular or treatment group was 69years and 68years in the controlled IV tPA group. This trial was an international, six-year trial, including patients in the US, Canada, Australia and Europe. The inclusion criteria consisted of AIS patients with an NIHSS $\geq 10$, anterior or posterior circulation, and $92 \%$ of the 306 patients with a baseline CTA had large vessel occlusions. The IV tPA group received standard of care treatment. In the endovascular group, IV tPA infusion was stopped at 40 minutes and the patient was taken to angiography for additional IA treatment via the following approved MT devices: Cocentric, Penumbra, Solitaire, infusion of IA tPA via Microsonic SV infusion system [EKOS] or standard microcatheter begun within 5hours and completed within 7hours of AIS symptom onset. Heparin was initiated with a 2000 unit IV bolus followed by an infusion at the rate of 450units/hour during the endovascular therapy. The Primary Outcome Measures for efficacy were analyzed using the Cochran-Mantel-Haenszel test utilizing a mRS of 0-2 at 90days (functional independence), adjusting for dichotomized baseline NIHSS strata and an $80 \%$ power to detect $10 \%$ difference between treatment approaches. The goal of this trial was to compare standard IV tPA treatment to endovascular therapy after IV tPA by evaluating disability-free survival at 90 days and symptomatic intracranial hemorrhage (ICH) within 30hours. The SYNTHESIS Expansion trial is a randomized, controlled, multicenter trial. ${ }^{12}$ This five-year trial enrolled 362 participants in Italy. The median age of the endovascular treatment group was 66years and 67years in the IV tPA group. The NIHSS score was not limited on the trial participants. Patients were required to have either anterior or posterior circulation. There is no data in regards to the percentage of patients with largeartery occlusions. The median time from the onset of AIS symptoms to treatment in the IV tPA arm was 2.75 hours and 3.75 hours in the endovascular treatment arm. The primary outcome was to assess disability-free survival at 90days (mRS: $0-2$ ) and powered to detect a $15 \%$ point advantage in the endovascular group. The two-tailed Fisher's exact test was used to compare dichotomized mRS results to the treatment strategy. The Mantel-Haenszel odds ratio was also performed with a $95 \%$ confidence interval in order to determine differences between the confounding variables. The Kaplan-Meier product limit method, followed by the log-rank test, was used to determine a difference in primary outcomes with a $95 \%$ confidence interval. The goal of the SYNTHESIS Expansion trial was to compare disability-free survival at 90days after treatment consisting of IV tPA within 4.5 hours of onset of AIS symptoms and endovascular treatment within 6hours of onset of AIS symptoms.

\section{Critique/Analysis}

The MR RESCUE trial utilized first generation MT devices with modest recanalization rates. Newer generation MT devices, such as the Covidien Solitaire Stent Retriever and Cocentric TREVO Stent Retriever, have demonstrated improved recanalization rates and lower complication rates ${ }^{13,14}$ There was a difference in predicted core volumes depending on if a patient was evaluated with a CT or MR imaging. The MR RESCUE trial outcomes were stratified by the presence of the penumbra. The authors defined penumbra as an infarct core less than $90 \mathrm{~mL}$ and a total ischemic tissue volume, or core, as less than or equal to $70 \%$ (minimum mismatch of $30 \%$ ). The groups with or without a measureable penumbra were not distinguished. The group in Table 1 who did not receive treatment with the Penumbra System showed a mismatch of $50 \%$. There was not a designated separation of penumbra and no penumbra, but rather into large and small infarct. The baseline $\mathrm{CT}$ and MRI images were used to determine penumbral patterns. However, the mean time to enrollment was 5.5 hours and time to groin puncture was 6.2 hours, during which time significant changes were likely to occur in the penumbral patterns. The patients with favorable penumbral patterns may have better functional outcomes regardless of which recanalization approach is implemented in the preferred time frame of $\leq 3$ hours from the onset of symptoms. This may be due to the quality of the collateral vessels. If the collateral vessels are of poor quality, then it may be theorized that the large vessel occlusion needs to be recanalized within minutes tohours for any chance of the patient having a good functional outcome. In patients with more vigorous collateral vessels, collateral blood flow may support the penumbral tissue longer allowing more time for spontaneous recanalization to 
occur. Thus, leaving the patient with an improved functional outcome. In MR RESCUE, revascularization was assessed at 7days. This time frame has been criticized as a clinically irrelevant monitoring plan for efficacy. However, it has been shown that infarct progression has been seen in patients with reperfusion, despite the absence of hemorrhagic transformation. ${ }^{15}$ Factors that may contribute to this are an undetermined duration, usually 1 to 2 hours, between the baseline scan and the opening of the artery and a further delay of 16 to 24 hours after the outcome angiogram during which unrecognized reocclusion would be possible. ${ }^{14}$ When surrogate outcomes were explored, it was found to be clinically relevant and universally applicable to obtain a 24 hour or 7day NIHSS in the assessment stroke trial outcomes. ${ }^{16,17}$ There is evidence that with increased prevalence of white matter hyperintensity (WMH), the risk of large and medium sized ischemic infarcts, microvascular infarcts, cerebral lacunar infarcts, and hemorrhagic strokes increase. ${ }^{18-20} \mathrm{WMH}$ has a linear relationship with increasing age and arterial hypertension. White matter injury (WMI) may be due to 3 potential pathophysiologic pathways: ionic, excitotoxic or oxidative stress pathways. These pathways all cause the white matter to become more vulnerable to ischemia. It is hypothesized that WMH may lead to a slow progressive ischemic change in the brain, almost a slow, chronic stroke. It is established that time is brain. Considering this, these chronic strokes would be resistant to thrombolytic therapy by the time the ischemic threshold was met to present severe symptoms. It would add to the explanation of why the elderly do not respond as well to tPA and have a greater risk of intracranial hemorrhage (ICH). WMH could, theoretically, also contribute to the quality of collateral vessels.

The studies that examined the relationship between diabetes mellitus (DM) and white matter disease (WMD) are contradictory. However, a recent large study found that elevated levels of HbAlc, a surrogate marker of hyperglycemia, had an association with increased WMD lesion load. ${ }^{21}$ The relationship between HbAlc was stronger than the DM diagnoses variable because some patients with prediabetes or impaired glucose control had HbAlc levels $>5.7 \%$, but were not diagnosed with DM. Conversely, patients were included with a DM diagnosis who had tightly controlled glucose levels yielding an $\mathrm{HbA} 1 \mathrm{c}<5.7 \%$. HbA1c is not thought to have a role in the development of cerebral microvascular changes. ${ }^{22}$ However, hyperglycemia does indirectly lead to microinfarctions and white matter lesions. ${ }^{23}$ Microinfarctions and white matter lesions may be explained by capillary thickening followed by vessel lumen narrowing and progressive ischemia in patients with WMD and DM. ${ }^{24}$ When metabolic changes and excitotoxicity were examined in infarct progression, glucose and pyruvate were both found to be lower in patients with delayed infarct progression. ${ }^{25}$ Initially, this was attributed to patients having a more intact metabolism. However, upon further study, it was also suggested that pyruvate has neuroprotective properties. ${ }^{26}$

Endothelial cell dysfunction in hyperglycemic states may also have a role in small vessel injury. ${ }^{27}$ Endothelial cells stiffen and soften in response to the sodium and potassium concentrations. This response is a determinant of nitric oxide release, which controls blood pressure in addition to the sodium influx affect on blood volume.

In addition, both $\mathrm{BNaC} 1$ and $\mathrm{BNaC} 2$ are two sodium channels found exclusively in the brain. ${ }^{28}$ The sodium channels are expressed primarily in the neurons, but are either absent or significantly decreased in white matter. The lack of these channels in WMD may account for the increased intracranial pressure (ICP). WMH may also be involved in the connection between hyponatremia and increased cerebral ischemia. ${ }^{29}$ The sodium channels are influenced by Thr594Met polymorphisms. In addition to this polymorphism having an obvious role in sodium regulation, it is also related to saltsensitive hypertension. The Thr594Met polymorphism occurs in 5\% of persons of African American descent. ${ }^{30}$ African Americans have a higher incidence of stroke than other ethnicities, nearly twice that of Caucasians. African Americans are also more likely to die from stroke than Caucasians. This is in part due to social and economic disparities, but may also be contributed to by these genetic differences. ${ }^{31}$

The authors of the MR RESCUE trial published another paper midway through the trial, which discussed the clinical evidence of the harmful effects of tPA. ${ }^{32}$ A GdDTPA-enhanced MRI was used to detect contrasted leakage into the subarachnoid space in order to measure blood brain barrier (BBB) integrity after AIS. Data was collected on 140 patients, 38 who were administered IA tPA, 24 who were administered IV tPA, 18 who had MT alone, and 60 who did not have any acute intervention. BBB breakdown was observed in $66 \%$ of the IA tPA treatment arm and $50 \%$ of the IV tPA arm, compared to $28 \%$ of the MT arm and $30 \%$ of the control arm ( $\mathrm{p}=0.002)$. It was concluded that thrombolysis with IPA, via either IA or IV tPA, was an independent predictor of BBB breakdown ( $\mathrm{p}=0.001)$, and BBB breakdown was an independent predictor of hemorrhagic transformation $(\mathrm{p}=0.007)$. The authors hypothesized that in addition to clot lysis, tPA may be directly affecting neurovascular function and integrity.

The IMS III trial has potential for bias in favoring the IV tPA group. There was not radiological confirmation of any occlusions included in the publication. Patients were included who were unlikely to benefit from IA tPA therapy, such as a significantly higher number of patients with brain stem or cerebellum strokes and higher NIHSS scores. In fact, the patient selection was performed based on NIHSS scores, expecting and finding that $20 \%$ of the patients enrolled did not have a large vessel occlusion. A thrombectomy can only be performed on a large vessel occlusion. Large vessel occlusions are inherently associated with worse outcomes compared to non-large vessel occlusions, which include peripheral branch occlusions and lacunar infarcts. This is important because distal and perforator occlusions have better response to IV tPA and often times resolve without any intervention. The selection bias created by including patients who were likely to have good outcomes regardless of treatment and patients who were not candidates for IA tPA therapy dilutes the results in both treatment arms and obscures the potential benefit. The significance of the selection bias was not included in the publication of the IMS III trial. However, Andrew Demchuk, MD presented data at the International Stroke Conference in 2013, which confirmed the significance. He analyzed the results in a subset of patients with the confirmed presence of a large vessel occlusion prior to randomization. In this subset of only patients who were eligible, endovascular treatment did show a benefit of $35 \%$ of patients achieving complete resolution of symptoms or neurologic recovery over IV tPA alone at $19.8 \%$ ( $p$-value $=0.0114)$. These results dispute the general conclusions of the IMS III trial. ${ }^{33}$ In the IMS III trial, the majority of patients were given a lower dose of IV tPA in the endovascular arm, only $2 / 3$ of the approved, standard of care dose, which could negatively impact the outcomes in this group. This protocol was amended in June 2011 and patients were given the standard IV tPA dose. There were 100 patients randomized to IA treatment $(24 \%)$ did not receive IA tPA after IV tPA for various reasons, including early clinical improvement or deterioration, absence of clot, and technical failures. 
The lesson repeated in stroke care is that "time is brain." The IMS trial series suggested that there is a $10 \%$ relative reduction in good outcomes for every 30-minute delay in endovascular treatment. Initiating IV tPA and then transferring a patient to a higher level of care facility is referred to as "drip and ship." There was an increase from $30 \%$ of patients in the IMS I trial to $70 \%$ of patients in the IMS III trial who "dripped and shipped," which subsequently led to a 32 minute increase in time to IA treatment. Another trial focused on the effect of faster onset to treatment times, broken down in 15 minute increments, showed an association with reduced in-hospital mortality (OR, 0.96; 95\% CI, 0.95-0.98; $\mathrm{P}<0.001$ ), reduced symptomatic ICH (OR, 0.96; 95\% CI, 0.95-0.98; $\mathrm{P}<0.001)$, increased achievement of independent ambulation at discharge (OR, 1.04; 95\% CI, 1.03-1.05; $\mathrm{P}<0.001)$, and increased discharged patients to home (OR, 1.03; 95\% CI, 1.02-1.04; $\mathrm{P}<.001){ }^{34}$ These statistics support the hypothesis that the negative results of the IMS III trial are likely due to delays in the transfer of patients rather that as intrinsic lack of benefit of IA therapy. The IMS III trial took place over a decade. During this time, endovascular care and technology has revolutionized. Current practice utilizes stent retrievers, which were not common in the IMS III trial. The IMS III trial did not record the use of in-line arterial air filters. The use of air filters for intracerebral use is varied among practitioners, but not uncommon. Recently, these filters have been found to absorb over $99 \%$ of tPA. ${ }^{35}$ Patients potentially treated with the use of these filters would not have received any therapeutic effect of the IA tPA.

The SYNTHESIS Expansion trial has some advantages over the IMS III trial. The patients in the SYNTHESIS Expansion trial had IA tPA therapy initiated sooner than the patients in the IMS III trial. The SYNTHESIS trial also had higher numbers of patients with large vessel occlusions and received more MT. However, the SYNTHESIS Expansion trial was also flawed. The authors do not report confirmation of large vessel occlusions, nor do they report how often large vessel occlusions were found on angiography. IA tPA was administered regardless of the presence of a large vessel occlusion. This practice is not the standard of care.

The SYNTHESIS Expansion trial did not limit the NIHSS score of the patients included in the trial. The NINDs study established that patients with higher NIHSS scores at the time of thrombolytic therapy have less benefit. ${ }^{1}$ There also was a lack of CT or MRI imagery. The median times to treatment varied between the IV tPA (2hours, 45minutes) and endovascular (3hours, 45 minutes) groups. The median time to IA tPA treatment was nearly one hour longer than the IV tPA. This discrepancy is important because recanalization effectiveness is highly dependent upon infarction development, which directly correlates to time.

In the SYNTHESIS Expansion trial, only $1 / 3$ of the patients were treated with a mechanical device. Therefore, the majority of comparisons were made between patients receiving systemic doses of tPA via IV or IA administration. It has been shown that high catheter doses of tPA are less effective than the lower doses. ${ }^{36}$ Hence, the IA protocol used in the SYNTHESIS Expansion trial was less effective than the same dose given IV. There was no follow-up or explanation for 15 patients in the endovascular arm who did not receive IA tPA. Although the outcomes of these patients were not individually reported, they were included in the overall outcomes for the IA treatment arm. The good outcomes were lower than expected at 30\%. This is especially significant given that the there was no exclusion criteria based on a minimum NIHSS score. There should have been a significant amount of patients who would have spontaneously improved, regardless of treatment. This suggests that the study flaws had a significant impact on the results. Complete revascularization rates achieved in practice are much higher than the MR RESCUE or IMS III trials. The Thrombolysis in Cerebral Infarction scale (TICI) was used to assess successful revascularization, with a range of 0 , indicating no perfusion, to 3 , indicating full perfusion. TICI $2 b$ or 3 revascularization, defined as $>50 \%$ of the affected territory, are associated with the highest rates of good outcomes (48\% and $78 \%$ respectively). ${ }^{37}$ The National INSTOR Registry reported $74 \%$ of patients achieving TICI $2 \mathrm{~b}$ or 3 in 136 patients. The MR RESCUE trial angiographic revascularization results showed that TICI $2 \mathrm{~b}$ or 3 was achieved in only $27 \%$ of the patients treated with the Penumbra device or embolectomy and $21 \%$ of those reported good outcomes. The good outcome results of the MR RESCUE were about half of what is seen in clinical practice, which is a reflection of the low success of angiographic revascularization. In the IMS III trial, TICI $2 \mathrm{~b}$ or 3 revascularization was only achieved in $40 \%$ of the endovascular group, $48 \%$ of which achieved good outcomes. For those patients comparable to the IMS III inclusion and exclusion criteria, INSTOR reports $56 \%$ achieving good outcomes using the same technology. TICI revascularization rates were not reported in the SYNTHESIS Expansion trial in order to compare the groups or effectiveness of the intervention. Although registry data has limited use in comparisons to trial data, this does suggest that the MR RESCUE and IMS III angiographic and clinical outcomes did not meet the standards of routine clinical care perhaps due to operator skill or selection bias. Since the publication of the trials, selection bias has been confirmed with the physicians involved with both of these trials.

Equipoise is a conceptual limitation applied to these three trials. ${ }^{38}$ Equipoise is difficult to define. Equipoise exists in situations with 2 or more competing possible treatment paradigms and a lack of definitive evidence upon which to conclude the best option for an individual patient. It involves balancing the interpretation of the results and requires the reliance on expert opinion. Specifically, equipoise in these trials relate to the predictors of outcome versus the response to therapy. High NIHSS scores and modified Rankin scores cannot be directly translated into a lack of response to therapy as compared to the standard of care. In addition, predictors of favorable outcomes, such as low NIHSS scores, modified Rankin scores, and good collateral flow, are not necessarily due to response to therapy. Poor outcomes are not always a result of choice of therapy in AIS. Poor outcomes are influenced by larger strokes in elderly patients, failure or delays to reperfusion, reperfusion after a cerebral infarct, and factors predisposing patients to hemorrhagic complications. The investigators in both the IMS III and SYNTHESIS Expansion trials both were limited by low recruitment rates. This was due to physician application of selection bias due to the FDA approval of certain devices and Medicare reimbursement. Clinician familiarity with the FDA approval processes of devices verses drugs is not always apparent. The high cost of new treatments and the tendency to prematurely terminate randomized controlled trials further complicates the equipoise and interpretation of the results of these trials.

\section{Summary of findings}

The three randomized, controlled trials presented in Table 1 failed to demonstrate that endovascular therapy with IA tPA is superior to IV tPA, but showed similar safety with IV tPA followed by endovascular therapy as compared to IV tPA alone. The MR RESCUE and IMS 
III trials both showed revascularization rates in the standard care of treatment groups that were higher than expected. This may be due to improved practices that have resulted through education, practice and the implementation of PSCs and CSCs, or the inclusion of large percentages of patients expected to spontaneously improve regardless of the intervention. The results from the three trials presented do not show endovascular treatment, including IA tPA, is superior to standard IV tPA treatment. However, these results lead to the exploration of new questions. Expert opinion does not support that these trials have proven endovascular treatment to be an inferior treatment approach in all patients. Patient selection is still questionable. Proposed selection criteria include the length of thrombus and the presence of the penumbra. ${ }^{39}$ IA tPA therapy is a specialty procedure offered at limited facilities. The transportation of patients to these facilities and the novelty of the prodcedure led to increased time to treatment. The evolution and development of superior MT devices may also impact the results of future trials. Given the established data on the strong relationship between the onset-to-needle time and the effectiveness of IV tPA, future trials of endovascular therapy must focus on minimizing delays to the initiation of endovascular therapy. There has not been a favorable penumbral pattern identified in order to establish which patients would benefit for endovascular therapy. Currently, alteplase or tPA, is the only FDA approved thrombolytic for the treatment of AIS. However, a recent study showed tenecteplase to be a superior thrombolytic for reperfusion, resulting in better clinical outcomes, as compared to alteplase. ${ }^{40}$ Future thrombolytic development and research may contribute to more conclusive outcomes.

Table I Evaluation of standard IV tpa verus endovascular treatment +/- IA tPA

\begin{tabular}{|c|c|c|c|c|c|c|c|c|c|}
\hline $\begin{array}{l}\text { Author(s) } \\
\text { Date/ } \\
\text { Ref \# }\end{array}$ & $\begin{array}{l}\text { Study } \\
\text { designs }\end{array}$ & $\begin{array}{l}\text { \# of } \\
\text { subjects }\end{array}$ & $\begin{array}{l}\text { Inclusion } \\
\text { criteria }\end{array}$ & $\begin{array}{l}\text { Exclusion } \\
\text { Criteria }\end{array}$ & $\begin{array}{l}\text { Study } \\
\text { location }\end{array}$ & $\begin{array}{l}\text { Study } \\
\text { duration }\end{array}$ & $\begin{array}{l}\text { Treatment } \\
\text { Regimens } \\
\text { evaluated }\end{array}$ & $\begin{array}{l}\text { Outcome } \\
\text { Variables } \\
\text { Measured \& } \\
\text { Method of } \\
\text { measurement }\end{array}$ & $\begin{array}{l}\text { Major findings } \\
\text { (Results) }\end{array}$ \\
\hline
\end{tabular}

\begin{tabular}{|c|c|c|c|c|c|c|c|c|c|}
\hline \multirow[t]{2}{*}{$\begin{array}{l}\text { Kidwell } \\
\text { CS. et } \\
\text { al." }\end{array}$} & \multirow[t]{2}{*}{ RCT } & \multirow[t]{2}{*}{$N=118$} & Age $18-85$ y/o & $\begin{array}{l}\text { NIHSS } \\
>1=30\end{array}$ & $\begin{array}{l}22 \text { sites } \\
\text { throughout } \\
\text { North } \\
\text { America }\end{array}$ & $\begin{array}{l}8 \text { years } \\
(2004- \\
2012)\end{array}$ & $\begin{array}{l}\text { All patients } \\
\text { pretreated } \\
\text { with CT or } \\
\text { MRI of brain } \\
\text { to determine } \\
\text { the presence } \\
\text { of a favorable } \\
\text { penumbral } \\
\text { pattern vs } \\
\text { non-penumbral } \\
\text { pattern during } \\
\text { randomization } \\
\text { (Favorable } \\
\text { penumbral } \\
\text { pattern was } \\
\text { defined as } \\
\text { a predicted } \\
\text { infarct core } \\
\text { of }<90 \mathrm{~mL} \& \\
\text { a proportion } \\
\text { of predicted } \\
\text { infarct tissue } \\
\text { within the at- } \\
\text { risk region of } \\
<70 \%\end{array}$ & $\begin{array}{l}\text { Primary } \\
\text { Outcome } \\
\text { Measures: } \\
\text { Efficacy. Modified } \\
\text { Rankin Score }\end{array}$ & $\begin{array}{l}\text { A favorable } \\
\text { penumbral } \\
\text { pattern on } \\
\text { neuroimaging } \\
\text { did not identify } \\
\text { patients } \\
\text { who would } \\
\text { differentially } \\
\text { benefit from } \\
\text { endovascular } \\
\text { therapy for } \\
\text { acute ischemic } \\
\text { stroke }\end{array}$ \\
\hline & & & $\begin{array}{l}\text { Patients } \\
\text { treated with } \\
\text { IV IPA without } \\
\text { successful } \\
\text { recanalization } \\
\text { were eligible } \\
\text { if MRA or } \\
\text { CTA showed } \\
\text { a persistent } \\
\text { large ocdusion }\end{array}$ & \multicolumn{3}{|c|}{ Contraindication to MRI (pacemaker) } & $\begin{array}{l}\text { Endovascular } \\
\text { Group: } \\
\text { Mechanical } \\
\text { embolectomy } \\
\text { (Merci } \\
\text { Retriever or } \\
\text { Penumbra } \\
\text { system) }\end{array}$ & $\begin{array}{l}\text { Secondary } \\
\text { Outcome } \\
\text { Measures: median } \\
\text { change in NIHSS } \\
\text { at 90days, Global } \\
\text { test statistic } \\
\text { at 90days, } \\
\text { hemorrhagic } \\
\text { transformation } \\
\text { at 7days, serious } \\
\text { ADRs at 90days, } \\
\text { mortality at } \\
90 \text { days }\end{array}$ & $\begin{array}{l}\text { Endovascular } \\
\text { treatment is } \\
\text { not superior to } \\
\text { standard IV IPA }\end{array}$ \\
\hline
\end{tabular}


Table Continued..

\begin{tabular}{|c|c|c|c|c|c|c|c|c|c|}
\hline $\begin{array}{l}\text { Author(s) } \\
\text { Date/ } \\
\text { Ref \# }\end{array}$ & $\begin{array}{l}\text { Study } \\
\text { designs }\end{array}$ & $\begin{array}{l}\text { \# of } \\
\text { subjects }\end{array}$ & $\begin{array}{l}\text { Inclusion } \\
\text { criteria }\end{array}$ & $\begin{array}{l}\text { Exclusion } \\
\text { Criteria }\end{array}$ & $\begin{array}{l}\text { Study } \\
\text { location }\end{array}$ & $\begin{array}{l}\text { Study } \\
\text { duration }\end{array}$ & $\begin{array}{l}\text { Treatment } \\
\text { Regimens } \\
\text { evaluated }\end{array}$ & $\begin{array}{l}\text { Outcome } \\
\text { Variables } \\
\text { Measured \& } \\
\text { Method of } \\
\text { measurement }\end{array}$ & $\begin{array}{l}\text { Major findings } \\
\text { (Results) }\end{array}$ \\
\hline
\end{tabular}

New focal

disabling

neurologic

deficit

consistent

with acute

cerebral

ischemia

(NIHSS 6-29)

Clot retrieval

procedure can

be initiated

within 8hours

from onset

Large vessel

proximal

anterior

circulation

occlusion on

MR or CT

angiography

(internal

carotid, MI or

M2 MCA)

$\mathrm{ICH}$

Standard medical care

Coma

Rapidly improving neurological signs prior to randomization

Pretreatment

MRI

performed

according to

MR RESCUE

protocol

Pre-existing medical, neurological or psychiatric disease that would psychiatric disease that would or imaging evaluations

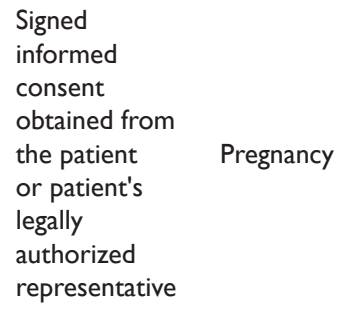

Signed

informed

consent

obtained from

the patient

or patient's

legally

authorized

representative

Pregnancy

Premorbid

modified

Rankin score

of 0-2
Known allergy to iodine previously refractory to pretreatment medications 
Table Continued..

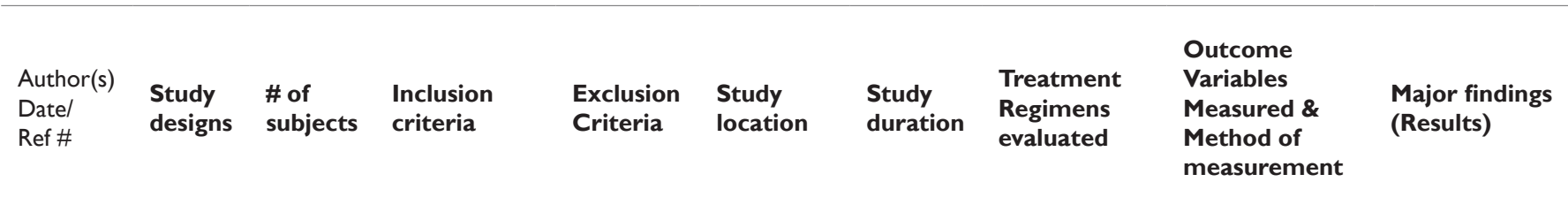

Allowed but
not required
patients
treated with
IV IPA up to
4.5 hours
from symptom
onset with
persistent
target
occlusion
on post-
treatment
MR RESCUE
MR or CT
treatment
MR RESCUE
MR or CT
completion of
drug infusion
(Note Rapidly
improving
neurological
signs prior to
randomization
is an
exclusion)

Contrast-Enhanced Neck MRA or CTA suggests proximal ICA occlusion, proximal carotid stenosis $>67 \%$, or dissection

INR $>3.0$

PTT $>3 \times$ Normal

Imaging data cannot be processed by the MR RESCUE computer

Renal Failure (serum creatinine $>20$ or Glomerular Filtraticn Rate [GFR] <30)

Contraindication to contrast dye (Hyperthyroidism, history of severe allergic reaction to iodinated contrast allergic reaction to iodinated contrast disease as an adult, including tumor or transplant surgery, or family history of kidney failure, paraproteinemia syndromes or multiple myeloma, collagen vascular disease, severe cardiac insufficiency, severely compromised liver function, current

therapy with metformin, aminoglycosides

\section{Clinical recommendation}

The IMS III, SYNTHESIS Expansion, and MR-RESCUE trials all contradicted the findings of the PROACT II trial that endovascular treatment is superior to IV tPA. It is uncertain whether the negative results of these trials are attributable to flaws in the study designs, outdated knowledge and technology, or whether there are fundamental reasons why endovascular therapy will never be superior to IV tPA. However, the flaws of the studies and consequent uncertainties in the conclusions have left an opportunity for future studies. The efficacy of
tPA was low due to the following factors: arterial pathology was not controlled and the trials included patients without arterial occlusions having excellent spontaneous prognosis, arterial recanalization and tissue perfusion was not achieved while ischemic brain tissue was still viable, and arterial recanalization did not prevent functional impairment because major portions of the ischemic territory were already irreversibly injured or side effects, like intracranial hemorrhage, impaired possible benefits. Leading neurologists have come to the consensus that there is still a benefit to endovascular therapy and that better patient selection would have yielded greater 
benefits. Edward C. Jauch, MD, from the Medical University of South Carolina in Charleston, commented, "This is in some ways, a call to arms for the healthcare system and stroke systems of care, to re-evaluate and be more introspective and evaluate these more severely affected stroke patients. I think it reinforces that tPA works in a significant portion of patients, and we should still consider that our standard of care regardless of stroke severity, but beyond that, we need to take a look at the larger strokes and try to find ways of being more efficient at getting them the definitive care that they may need, which was alluded to in the IMS III results, that the larger strokes had the greatest suggestion of benefit". ${ }^{41}$ The AHA/ASA updated their guidelines in 2013 after the release of these studies. They currently support the recommendation that IA fibrinolysis is beneficial for the treatment of carefully selected patients with major ischemic strokes less than 6hours in duration caused by occlusions of the MCA who are not otherwise candidates for IV tPA (Class I; Level of Evidence B). The optimal dose of IA tPA is not well established, and tPA administered via the IA route does not have the FDA approval. ${ }^{42}$ However, the embolectomy devices are approved by the FDA. Once the FDA approved the devices and Medicare provided reimbursement for the procedures, endovascular treatment became widespread and many physicians felt that the answer was in. Thus, diminishing equipoise. The use of endovascular treatment has nearly tripled since $2009.43,44$

\section{Conclusion}

The IMS III and SYNTHESIS Expansion trials demonstrated that the first-line treatment of AIS should continue to be IV tPA within 4.5 hours of symptom onset, regardless of major intracranial artery occlusion on CTA or MRI imagery and despite higher revascularization rates in the endovascular treatment groups. The MR RESCUE trial did not provide evidence in support of implementing endovascular treatment beyond 4.5hours of onset of AIS symptoms in patients, regardless of their penumbral pattern or size. However, the flaws in these trials raise questions on the clinical applicability of the conclusions. The standard of care, IV tPA therapy, has the benefits of speed and convenience with presumed lower rates of recanalization of large artery occlusions as compared to IA methods. Higher rates of recanalization were demonstrated in more recent reports of major arterial occlusions treated with IV t-PA, as measured by transcranial Doppler and magnetic resonance angiography. Conversely, IA therapies report higher recanalization rates, but are dependent on many inconsistent factors, such as procedural delays and risks, and even failing to be applied in patients where time to reperfusion remains to be a critical factor. Higher rates of recanalization in IA trials using clot-removal devices have not translated into improved patient functional outcomes as compared to trials of IV therapy. Combined IV-IA therapy provides synergistic benefits of both, but only when applied without the time delays seen in practice, which may or may not improve, as the systems evolve concerning this novel practice. Where equipoise exists, randomizing appropriate patients to either IV tPA therapy or IV tPA followed by IA tPA, while incorporating current technologies in terms of mechanical devices, is a rational and appropriate approach.

\section{Acknowledgements}

None.

\section{Conflict of interest}

Author declares that there is no conflict of interest.

\section{References}

1. Tissue plasminogen activator for acute ischemic stroke. The National Institute of Neurological Disorders and Stroke rt-PA Stroke Study Group. N Engl J Med. 1995;333(24):1581-1587.

2. Hacke W, Kaste M, Bluhmki E, et al. Thrombolysis with alteplase 3 to 4.5 hours after actue ischemic stroke. $N$ Engl $\mathrm{J}$ Med. 2008;359(13):1317-1329.

3. Rha JH, Saver JL. The impact of recanalization on ischemic stroke outcome: a meta-analysis. Stroke. 2007;38(3):967-973.

4. Furlan A, Higashida R, Wechsler L, et al. Intr-arterial prourokinase for acute ischemic stroke - the PROACT II study: a randomized controlled trial. JAMA. 1999;282(21):2003-2011.

5. Smith WS, Sung G, Starkman S, et al. Safety and efficacy of mechanical embolectomy in acute ischemic stroke: results of the MERCI trial. Stroke. 2005;36(7):1432-1438.

6. Penumbra Pivotal Stroke Trial Investigators. The Penumbra Pivotal Stroke Trial: safety and effectiveness of a new generation of mechanical devices for clot removal in intracranial large vessel occlusive disease. Stroke. 2009;40(8):2761-2768.

7. Lewandowski CA, Frankel M, Tomsick TA, et al. Combined intravenous and intra-arterial r-TPA versus intra-arterial therapy of acute ischemic stroke: Emergency Management of Stroke (EMS) Bridging Trial. Stroke. 1999;30(12):2598-2605.

8. Broderick JP, Palesch YY, Demchuk AM, et al. Endovascular Therapy after Intravenous t-PA verses t-PA Alone for Stroke (IMS III Trial). $N$ Engl J Med. 2013;368(10):893-903.

9. Broderick JP. The Interventional Management of Stroke (IMS II Trial). Stroke. 2007;38(7):2127-2135.

10. Khatri P, Neff J, Broderick JP. Combined Intravenous and Intra-Arterial Recanalization for Acute Ischemic Stroke: The Interventional Management of Stroke Study (IMS-I Trial). Stroke. 2004;35(4):904-911.

11. Kidwell CS, Jahan R, Gornbein J, et al. A Trial of Imaging Selection and Endovascular Treatment for Ischemic Stroke (MR RESCUE trial). $N$ Engl J Med. 2013;368(10):914-923.

12. Ciccone A, Valvassori L, Nichelatti M, et al. Endovascular Treatment for Acute Ischemic Stroke (SYNTHESIS Expansion Trial). $N$ Eng $J$ Med. 2013;368(10):904-913.

13. Nogueira RG, Lutsep HL, Gupta R, et al. Trevo versus Merci retrievers for thromboectomy revascularisation of large vessel occlusions in acute ischaemic stroke (TREVO 2): a randomised trial. Lancet. 2012;380(9849):1231-1240.

14. Saver JL, Jahan R, Levy EI, et al. Solitaire flow restoration device versus the Merci retriever in patients with acute ischaemic stroke (SWIFT): a randomised, parallel-group, non-inferiority trial. Lancet. 2012;380(9849):1241-1249.

15. Lees KR. Serial Alberta Stroke Program Early Computed Tomography Score from baseline to 24 hours in SWIFT: a novel surrogate end point for revascularization in acute stroke. Stroke. 2014;45(3):653-654.

16. Broderick JP, Lu M, Kothari R, et al. Finding the most powerful measures of effectiveness of tissue plasminogen activator in the NINDS tPA stroke trial. Stroke. 2000;31(10):2335-2341. 
17. Kerr DM, Fulton RL, Lees KR, et al. Seven-day NIHSS is a sensitive outcome measure for the exploratory trials in acute stroke: evidence from the Virtual International Stroke Trials Archive. Stroke. 2012;43(5):1401-1403.

18. Snowdon DA, Greiner LH, Mortimer JA, et al. Brain infarction and the clinical expression of Alzheimer Disease: the Nun study. JAMA. 1997;277(10):813-817.

19. Brundel M, de Bresser J, van Dillen JJ, et al. Cerebral microinfarcts: a systematic review of neuropathological studies. J Cereb Blood Flow Metab. 2012;32(3):425-436.

20. Vermeer SE, Longstreth WT, Koudstaal PJ. Silent brain infarcts: a systemic review. Lancet Neurol. 2007;6(7):611-619.

21. Rozanski M, Richter TB, Grittner U, et al. Elevated levels of hemoglobin A1c are associated with cerebral white matter disease in patients with stroke. Stroke. 2014;45(4):1007-1011.

22. Inzucchi SE. Clinical practice. Diagnosis of diabetes. $N$ Eng $J$ Med. 2012;367(6):542-550.

23. Gold G, Kövari E, Herrmann FR, et al. Cognitive consequences of thalamic, basal ganglia, and deep with matter lacunes in brain aging and dementia. Stroke. 2005;36(6):1184-1188.

24. Brown WR, Thore CR. Review: cerebral microvascular pathology in ageing and neurodegeneration. Neuropathol Appl Neurobiol. 2011;37(1):56-74.

25. Woitzik J, Pinczolits A, Hecht N, et al. Excitotoxicity and metabolic changes in association with infarct progression. Stroke. 2014;45(4):1183-1185.

26. Ryou MG, Liu R, Ren M, et al. Pyruvate protects the brain against ischemia-reperfusion injury by activating the erythropoietin signally pathway. Stroke. 2012;43(4):1101-1107.

27. Onat D, Brillon D, Colombo PC, et al. Human vascular endothelial cells a model system for studying vascular inflammation in diabetes and atherosclerosis. Curr Diab Rep. 2011;11(3):193-202.

28. García-Añoveros J, Derfler B, Neville-Golden J, et al. BNaC1 and $\mathrm{BNaC} 2$ constitute a new family of human neuronal sodium channels related to degenerins and epithelial sodium channels. Proc Natl Acad Sci USA. 1997;94(4):1459-1464.

29. Hasan D, Wijdicks EF, Vermeulen M. Hyponatremia is associated with cerebral ischemia in patients with aneurysmal subarachnoid hemorrhage. Ann Neurol. 1990;27(1):106-108.

30. Baker EH, Dong YB, Sagnella GA, et al. Association of hypertension with $T 594 M$ mutation in beta subunit of epithelial sodium channels in black people resident in London. Lancet. 1998;351(9113):1388-1392.
31. Go AS, Mozaffarian D, Roger VL, et al. Heart disease and stroke statistics-2014 update: a report from the American Heart Association. Circulation. 2014;129(3):e28-e292.

32. Kidwell CS, Latour L, Saver JL, et al. Thrombolytic toxicity: blood brain barrier disruption in human ischemic stroke. Cerebrovac Dis. 2008;25(4):338-343

33. Demchuk A. IMS III: Comparison of Outcomes between IV and IV/IA Treatment in Baseline CTA Confirmed ICA, M1, M2 and Basilar Occlusions, slide 20, Presented at ISC, Honolulu, Hawaii, USA; 2013.

34. Saver JL, Fonarow GC, Smith EE, et al. Time to treatment with intravenous tissue plasminogen activator and outcome acute ischemic stroke. JAMA. 2013;309(23):2480-2488.

35. Stroke Treatment Dispute-SIR Position Paper Responds to Perceived Threat Against Intra-Arterial Ischemic Stroke Revascularization. Radiology Today. 2013;14(11):28.

36. Bookstein JJ, Bookstein FL. Augmented experimental pulse-spray thrombolysis with tissue plasminogen activator, enabling dose reduction by one or more orders of magnitude. J Vasc Interv Radiol. 2000;11(3):299-303.

37. Interventional Stroke Theraphy Outcomes Registry. Empowering Data-Driven Stroke Treatment.

38. Tomsick TA, Khatri P, Jovin T, et al. Equipoise among recanalization strategies. Neurology. 2010;74(13):1069-1076.

39. Riedel $\mathrm{CH}$, Zimmermann P, Jensen-Kondering $\mathrm{U}$, et al. The importance of size: Successful recanalization by intravenous thrombolysis in acute anterior stroke depends on thrombus length. Stroke. 2011;42(6):1775-1777.

40. Parsons M, Spratt N, Bivard A, et al. A randomized trial of tenecteplase versus alteplase for acute ischemic stroke. $N$ Engl J Med. 2012;366(12):1099-1107.

41. Jeffrey S. IMS III: No benefit of endovascular therapy after thrombolysis. 2014.

42. Jauch EC, Saver JL, Adams HP, et al. Guidelines for the early management of patients with acute ischemic stroke: A guideline for healthcare professionals from the American Heart Association/American Stroke Association. Stroke. 2013;44(3):870-947.

43. Hassan AE, Chaudhry SA, Grigoryan M, et al. National trends in utilization and outcomes of endovascular treatment of acute ischemic stroke patients in the mechanical thrombectomy era. Stroke. 2012;43(11):3012-3017.

44. Zaidat OO, Lazzaro M, Mc Ginley E, et al. Demand-supply of neurointerventionalists for endovascular ischemic stroke therapy. Neurology. 2012;79(13 Suppl 1):S35-S41. 